\title{
Facteurs physiques contrôlant la photoréactivité de polymères à groupes pendants de type cinnamique
}

\author{
X. Coqueret
}

\author{
Laboratoire de Chimie Macromoléculaire, URA 351 du CNRS, USTL, 59655 Villeneuve-d'Ascq \\ cedex, France
}

\begin{abstract}
Résumé : Les polymères linéaires à groupes pendants dérivés de l'acide cinnanique sont le siège de réactions photo-induites de dimérisation assurant le pontage des chaînes et causant leur réticulation. La réactivité de plusieurs séries de polymères photosensibles originaux, à chaîne principale de type silicone ou de type polyvinylamine modifiée, en solution ou sous forme de film à l'état condensé, a été examinée à l'aide de la spectroscopie UV. Les données cinétiques ont été exploitées pour révéler des comportements spécifiques à chacune des séries. Le rendement quantique de dimérisation et l'importance relative du processus compétitif de photo-isomérisation dépendent en particulier de facteurs physiques caractéristiques de la structure du polymère.
\end{abstract}

\section{INTRODUCTION}

Le poly(cinnamate de vinyle) PVCin est le précurseur et le représentant possédant la structure la plus simple des polymères synthétiques réticulables sous l'effet d'une exposition au rayonnement ultraviolet [1]. Le mécanisme principal conduisant à la perte de la solubilité du polymère est l'établissement de pontages covalents entre les chânes initialement linéaires par dimérisation des groupes cinnamates pendants. On se réfère assez souvent à la photodimérisation qui se produit dans des composés éthyléniques de faible masse molaire irradiés à l'état cristallin [2]. La réaction se produit sous strict contrôle géométrique de la maille cristalline, seules les formes présentant l'orientation et les distances intermoléculaires convenables pouvant conduire au dimère cyclobutanique par photocycloaddition [2+2].

La transposition de ce concept de la topochimie issu de l'examen des réactions au sein du cristal à des chromophores de même nature mais portés par des polymères donne une vision qualitativement acceptable du phénomène. Il faut cependant prendre en compte l'état amorphe du PVCin et de ses analogues dans les conditions habituelles du traitement photochimique. Ainsi la réactivité de chromophores doit révéler l'influence de paramètres physiques caractérisant la disposition relative des chromophores réactifs et leur mobilité dans la matrice.

L'intérêt de la question n'est pas limité à l'aspect fondamental des relations structure - photoréactivité, cette famille de polymères photosensibles ayant été développée initialement pour des applications photolithographiques [3]. Dans le contexte de nouvelles applications, l'étude approfondie de la réactivité de chromophores cinnamiques liés à des polymères d'état physique et de morphologie bien différenciés doit permettre une meilleure approche de la maitrise des réactions, en termes de nature et de cinétique.

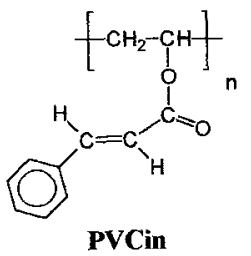

Cette étude porte sur une série de polymères dérivés de la polyvinylamine (PVAm-Cin) [4] et sur deux séries de polysiloxanes linéaires (PS-Cin [5], PS-CSA [6]) dont les caractéristiques chimiques principales sont représentées dans les trois formules ci-après. Dans chaque série, les différents polymères se caractérisent par le nombre moyen d'unités de répétition et par la proportion d'unités portant un chromophore $(p / n+p)$, cinnamique pour PVAm-Cin et PS-Cin, ou son homologue cyanostyrylacrylique pour PS-CSA.

\section{METHODES ET MODELES}

\subsection{Isomérisation des dérivés de l'acide cinnamique}

Lorsque les chromophores cinnamiques sont irradiés en solution diluée, la probabilité d'accomplissement 


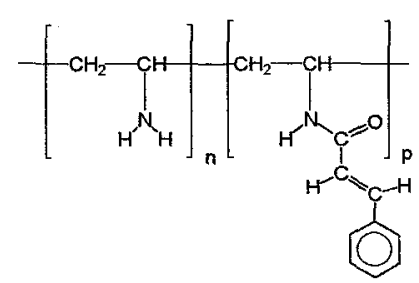

PVAm-Cin

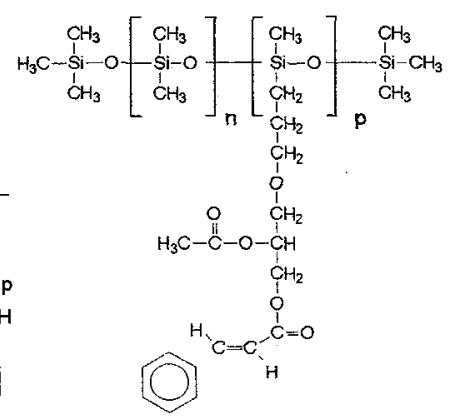

PS-Cin

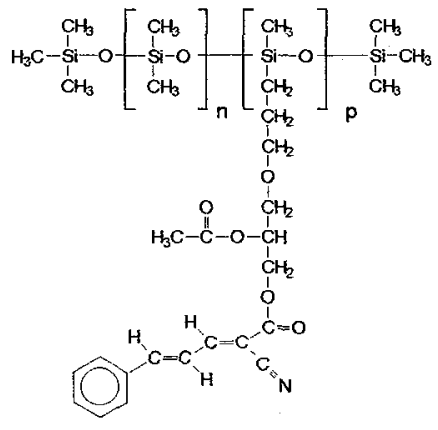

PS-CSA

de la réaction intermoléculaire de dimérisation pendant la durée de vie de l'état excité devient très faible. Le chromophore est alors le siège d'une interconversion entre isomères de configuration $\mathrm{E}$ et $\mathrm{Z}$ (Schéma 1). On observe en suivant l'évolution des spectres UV des modèles soumis à l'irradiation l'apparition d'un point isobestique, comme celui qui apparaît Figure 1 pour le $\mathrm{N}$-isopropyl cinnamamide de configuration initiale $\mathrm{E}$ exclusivement. Il s'établit par irradiation à $312 \mathrm{~nm}$ un état stationnaire dont la composition est déterminée par le rapport des intensités lumineuses absorbées par chacune des deux formes et par leurs rendements quantiques d'interconversion.

Lorsque des solutions de PVAm-Cin sont soumises à ce traitement, leurs spectres UV évoluent globalement de la même façon, mais sans présenter le point isobestique à $250 \mathrm{~nm}$. Ce comportement indique la disparition d'au moins une des deux formes isomères et amène à prendre en compte la réaction compétitive de dimérisation, rendue possible par l'existence d'une prédisposition favorable pour une fraction de chromophores voisins le long d'une même chaîne. Lorsque l'espaceur reliant les chromophores dimérisables à la chaîne principale est plus long, comme dans les polymères PS-Cin et PS-CSA, la dilution joue l'effet attendu, conduisant au processus monomoléculaire d'isomérisation uniquement. On observe avec ce type de polymères une évolution spectrale semblable à celle observée pour leurs modèles de faible masse molaire placés dans les mêmes solvants avec un point isobestique correspondant à la longueur d'onde d'iso-absorption des deux formes isomères.

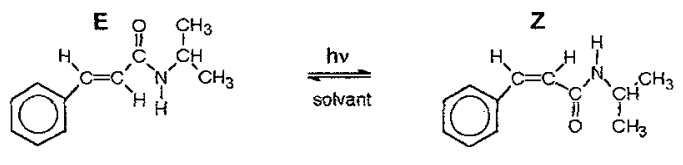

Schéma 1. Isomérisation du $\mathrm{N}$-isopropyl cinnamamide

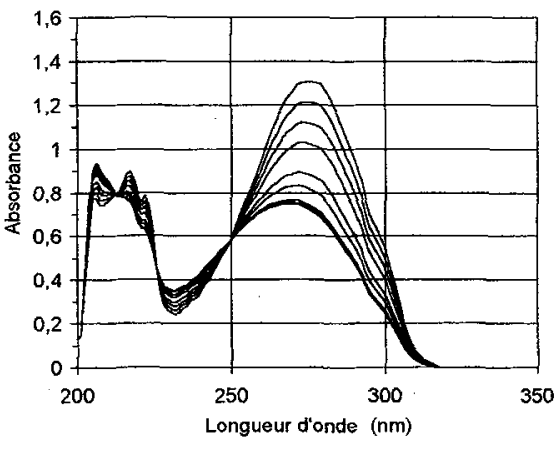

Figure 1. Suivi par spectroscopie UV de l'irradiation à $312 \mathrm{~nm} \mathrm{du} \mathrm{N}$-isopropyl cinnamamide $53 \mu \mathrm{mol} .1^{-1}$ dans $u$ mélange eau-THF $30 / 70 \mathrm{v} / \mathrm{v}$.

\subsection{Propriétés spectroscopiques de dérivés cinnamiques modèles}

La connaissance des caractéristiques spectroscopiques des différentes formes isomères en présence, position des maximums $\left(\lambda_{\max }^{E}, \lambda_{\max }^{2}\right)$ et du point isobestique pour l'isomérisation $\left(\lambda_{i s o}\right)$, de la valeur des coefficients d'absorption molaires $\varepsilon$ de ces espèces aux longueurs d'onde remarquables permet de déterminer à partir des valeurs de l'absorbance mesurées à $\lambda_{\max }^{E}$ et à $\lambda_{i s o}$, l'évolution des différentes formes isomères $E, Z$, ou dimérisée $D$ du chromophore cinnamique (Equations 1-3). Cela suppose néanmoins que seuls les événements décrits dans le Schéma 2 prennent place sous l'effet de l'irradiation. 


$$
\frac{[E]_{i}}{[E]_{0}}=\frac{\varepsilon_{\lambda_{\max }^{E}}^{E}}{\varepsilon_{\lambda_{\max }^{E}}^{E}-\varepsilon_{\lambda_{\max }^{E}}^{Z}}\left[\frac{A_{\lambda_{\max }^{E}}^{t}}{A_{\lambda_{\max }^{E}}^{0}}-\frac{\varepsilon_{\lambda_{\max }^{E}}^{Z} \cdot A_{\lambda_{i s o}}^{t}}{\varepsilon_{\lambda_{\max }^{E}}^{E} \cdot A_{\lambda_{\text {iso }}}^{0}}\right] \frac{[Z]_{i}}{[E]_{0}}=\frac{\varepsilon_{\lambda_{\max }^{E}}^{E}}{\varepsilon_{\lambda_{\max }^{E}}^{E}-\varepsilon_{\lambda_{\max }^{E}}^{Z}}\left[\frac{A_{\lambda_{\max }^{E}}^{t}}{A_{\lambda_{\max }^{E}}^{0}}-\frac{A_{\lambda_{i s o}}^{t}}{A_{\lambda_{i s o}}^{0}}\right] \frac{[D]_{i}}{[E]_{0}}=1-\frac{A_{\lambda_{i s o}}^{t}}{A_{\lambda_{i s o}}^{0}}
$$

Equ. 1

Equ. 2

Equ. 3

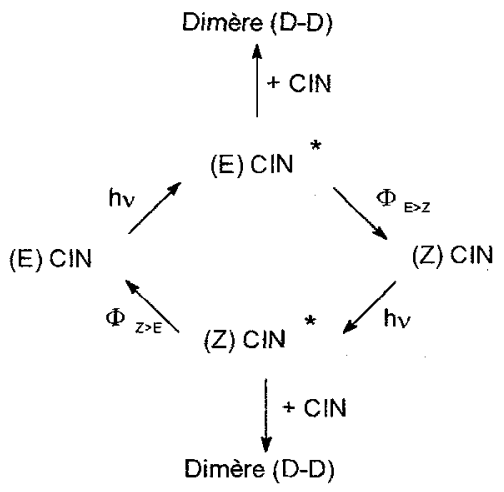

Tableau 1. Caractéristiques spectroscopiques des dérivés cinnamiques.

\begin{tabular}{|c|c|c|c|c|c|}
\hline Type & Solvant & $\begin{array}{c}\lambda_{\max } \\
\mathrm{nm}\end{array}$ & $\begin{array}{c}\lambda_{\text {iso }} \\
\mathrm{nm}\end{array}$ & $\begin{array}{c}\varepsilon \dot{\mathrm{a}} \lambda_{\max }^{Z} \\
1 / \mathrm{mol} . \mathrm{cm}\end{array}$ & $\begin{array}{c}\varepsilon \grave{a ̀ ~} \lambda_{\max }^{E} \\
1 / \text { mol.cm }\end{array}$ \\
\hline ester $^{\mathrm{a})} \mathrm{E}$ & éthanol & 276 & 250 & - & 21200 \\
\hline ester $^{\mathrm{a})} \mathrm{Z}$ & éthanol & - & 250 & - & 9300 \\
\hline amide $^{(\mathrm{b})} \mathrm{E}$ & Eau-THF 80/20 & 274,5 & 250 & 14000 & 24600 \\
\hline amide $^{\mathrm{b}} Z$ & Eau-THF 80/20 & 253 & 250 & 11200 & 5950 \\
\hline amide $^{\mathrm{b})} \mathrm{E}$ & Eau-THF 30/70 & 271,5 & 247,5 & 18200 & 24700 \\
\hline amide $^{\mathrm{b}} Z$ & Eau-THF 30/70 & 258 & 247,5 & 14000 & 9400 \\
\hline
\end{tabular}

a) cinnamate d'éthyle

b) N-isopropyl cinamamide

Schéma 2. Compétition entre isomérisation des formes $Z$ et $E$ et dimérisation des chromophores cinnamiques photo-irradiés.

L'étude de modèles des chromophores irradiés en solution diluée permet de déterminer les caractéristiques spectroscopiques recherchées. Lorsque ces dernières dépendent de la polarité du milieu et des associations intermoléculaires, il est nécessaire de reproduire en solution diluée un environnement comparable à celui de l'état condensé. Ainsi, pour la série PVAm-Cin et son modèle N-isopropyl cinnamamide, des solutions eau-tétrahydrofurane (THF) de composition variable ont fait l'objet d'une étude détaillée qui a permis de déterminer les valeurs rassemblées dans le Tableau 1 [7]. Pour les esters cinnamiques dont les propriétés à l'état condensé sont proches de celles déterminées en solution dans les solvants organiques courants, les valeurs généralement admises dans la littérature se sont révélées convenables. Le comportement particulier des polymères PS-CSA, comme on le verra plus bas, ne nécessite pas la connaissance des caractéristiques des formes isomérisées du chromophore [5].

\section{RESULTATS ET DISCUSSION}

L'examen des changements dans les spectres UV des trois familles de polymères irradiés à l'état condensé à l'aide de rayonnement UV non cohérents peu polychromatiques $(\Delta \lambda \approx 20 \mathrm{~nm})$, et exempts d'infrarouge pour éviter la perturbation des phénomènes étudiés, révèle des réactivités bien différenciées.

\subsection{Photoréactivité à l'état condensé des polymères PS-CIN et PVAm-CIN}

Les polymères PS-Cin sont des liquides visqueux et amorphes à température ambiante qui réticulent assez efficacement dans ces conditions par dimérisation des groupes pendants [5]. La dose d'énergie conduisant à la perte de solubilité par gélification diminue lorsque la masse molaire du polymère initial et (ou) lorsque la teneur en groupes cinnamiques dans le polymère $\mathbf{p} / \mathbf{n}+\mathbf{p}$ augmentent. Le suivi par spectroscopie UV de la photoréactivité d'un polymère PS-Cin de fonctionnalité maximale $(n=0)$ donne le profil de conversion représenté sur la Figure 2 a). Le rendement quantique de dimérisation $\Phi_{\text {dim, }}$ proportionnel à la pente $\Delta[\mathrm{D}] /[\mathrm{E}]_{0} . \Delta \mathrm{t}$ pour des films peu absorbants, est au moins 10 fois plus élévé que le rendement quantique d'isomérisation $\mathrm{E}^{*} \rightarrow \mathrm{Z}$. Le volume libre et les faibles associations conduisent à une faible densité instantanée de paires présentant la géométrie favorable à la dimérisation. La mobilité élevée permet cependant la formation de paires de collision pouvant évoluer vers la forme dimérisée $\mathrm{D}$ dont la fraction molaire maximale approche 0,6. Les polymères PVAm-Cin sont des solides vitreux, les rendements quantiques $\Phi_{\text {dim }}$ et $\Phi_{\text {isom }}$ sont initialement peu différents pour un échantillon donné (faible volume libre, peu d'associations favorables). Les polymères présentent une dose de gel dépendant non seulement des paramètres de composition vus plus haut [4], mais aussi de l'état d'ionisation des fonctions amine qui peuvent exister à l'état neutre ou sous forme ammonium. 


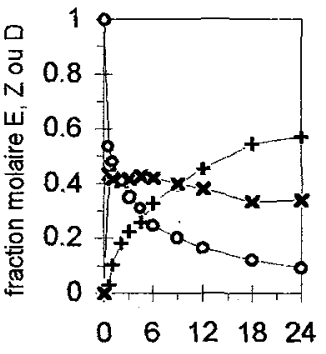

a)

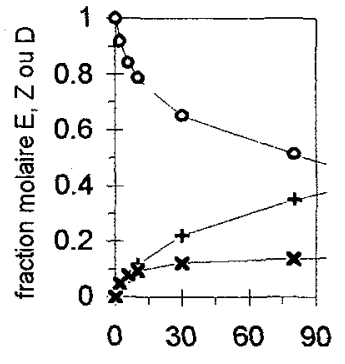

temps d'irradiation (s)

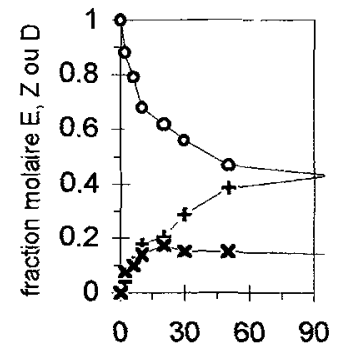

temps d'irradiation (s)

Figure 2. Profils de conversion des chromophores $E(O), Z(X)$ et dimérisés $D(+)$ sous irradiation à $254 \mathrm{~nm}$ d'un film de PSCin $([\mathrm{Cin}]=3 \mathrm{mmol} / \mathrm{g})$ irradié à $300 \mathrm{~nm}$ a), de PVAm-Cin sous forme neutre b), ou cationique $\mathrm{c})(\mathrm{p} / \mathrm{n}+\mathrm{p}=0,3)$ irradié à $254 \mathrm{~nm}$.

Les profils de conversion $b$ et $c$ de la Figure 2 enregistrés pour des films solides indiquent que la valeur plus faible de la dose de gel pour les PVAm-Cin irradiés sous forme cationique provient au moins en partie de la plus grande prédisposition des chromophores pour la dimérisation dans l'environnement cationique. Ces résultats ont été confirmés par la mesure et la comparaison des valeurs de $\Phi_{\text {dim }}$ pour des compositions $\mathrm{p} / \mathrm{n}+\mathrm{p}$ variées et pour différentes longueurs d'onde d'irradiation [8]

\subsection{Photoréactivité à l'état condensé des polymères PS-CSA}

Les polymères PS-CSA présentent une séparation de phases entre les groupes latéraux photosensibles rigides et polaires et la chaîne principale. Cette nature hétérogène se traduit par plusieurs caractéristiques remarquables révélées par la spectroscopie UV. L'isomérisation n'est pas observée à l'état condensé et le rendement quantique initial $\Phi_{\mathrm{dim}, 0}$ n'est pas dépendant du rapport $\mathbf{p} / \mathbf{n}+\mathbf{p}$. Il évolue avec l'avancement de la réaction selon une loi $\Phi_{\mathrm{dim}}=\Phi_{\mathrm{dim}, 0} . \alpha^{3}$ (avec $\left.\alpha=[\mathrm{CSA}]_{\mathfrak{t}} /[\mathrm{CSA}]_{0}\right)$ semblable à celle de polymères amorphes. L'état d'organisation mésomorphe observé semble réduire le volume libre et interdire l'isomérisation sans toutefois présenter les caractéristiques cinétiques attendues pour un système cristallin $[6,9]$.

\section{CONCLUSION}

L'étude par spectroscopie UV des photoréactions induites dans des polymères à groupes pendants de type cinnamique s'avère particulièrement utile pour analyser les profils de conversion et quantifier la cinétique des divers processus mis en jeu. Les trois exemples présentés montrent en particulier comment l'état physique de trois polymères contrôle la compétition isomérisation - dimérisation. La maitrise de ces réactions en vue d'applications variées, photolithographie, couches d'alignement pour cristaux liquides, immobilisation de dopants dans des films organiques, peut être réalisée par le choix du type de polymère photosensible et des caractéristiques du rayonnement utilisé pour le traitement photochimique [10].

\section{Références}

1. P. L. Egerton, E. Pitts, A. Reiser, Macromolecules 14, 95 (1981)

2. V. Enkelmann, G. Wegner, K. Novak, K. B. Wagener, J. Am. Chem. Soc. 115, 10390 (1993)

3. L. Minsk, J. G. Smith, W. P. Van Deusen, J. F. Wright, J. Appl. Polym. Sci. 2, 302 (1959)

4. A. El Achari, X. Coqueret, J. Polym. Sci. Part A: Polym. Chem. 35, 2513 (1997)

5. A. Hajaiej, X. Coqueret, A. Lablache-Combier, C. Loucheux, Makromol. Chem. 190, 327 (1989)

6. X. Coqueret, A. El Achari, A. Hajaiej, A. Lablache-Combier, C. Loucheux, L. Randrianarisoa, Makromol. Chem. 192, 1517 (1991)

7. A. El Achari, X. Coqueret, soumis pour publication

8. X. Coqueret, J. Photochem. Photobiol., Chemistry, 115, 143 (1998)

9. J. M. Buisine, X. Coqueret, C. Lahamamssi, Mol. Cryst. Liq. Cryst. 281, 295 (1996)

10. X. Coqueret, Macromol. Chem. Phys. à paraitre (1999) 